\title{
Fabrics coated with lubricated nanostructures display robust omniphobicity
}

\section{Citation}

Shillingford, Cicely, Noah MacCallum, Tak-Sing Wong, Philseok Kim, and Joanna Aizenberg. 2013. Fabrics Coated with Lubricated Nanostructures Display Robust Omniphobicity. Nanotechnology 25, no. 1: 014019. doi:10.1088/0957-4484/25/1/014019.

\section{Published Version}

doi:10.1088/0957-4484/25/1/014019

\section{Permanent link}

http://nrs.harvard.edu/urn-3:HUL.InstRepos:27657492

\section{Terms of Use}

This article was downloaded from Harvard University's DASH repository, and is made available under the terms and conditions applicable to Open Access Policy Articles, as set forth at http:// nrs.harvard.edu/urn-3:HUL.InstRepos:dash.current.terms-of-use\#OAP

\section{Share Your Story}

The Harvard community has made this article openly available.

Please share how this access benefits you. Submit a story.

Accessibility 
Fabrics coated with lubricated nanostructures display robust omniphobicity

\title{
Cicely Shillingford ${ }^{1 \dagger}$, Noah MacCallum ${ }^{1 \dagger}$, Tak Sing Wong ${ }^{1}$, Philseok Kim ${ }^{1}$, Joanna Aizenberg ${ }^{1 *}$
}

School of Engineering and Applied Sciences, Wyss Institute for Biologically Inspired Engineering and Kavli Institute for Bionano Science and Technology, Harvard University, Cambridge, Massachusetts 02138, USA

$\dagger$ Equal Contribution *Corresponding Author: jaiz@seas.harvard.edu

\section{Keywords}

nanostructured coating, slippery liquid-infused porous surfaces, omniphobicity, self-cleaning, fabrics

\begin{abstract}
The development of a stain-resistant and pressure-stable textile is desirable for consumer and industrial applications alike, yet it remains a challenge that current technologies have been unable to fully address. Traditional superhydrophobic surfaces, inspired by the lotus plant, are characterized by two main components: hydrophobic chemical functionalization and surface roughness. While this approach produces water-resistant surfaces, these materials have critical weaknesses that hinder their practical utility, in particular as robust stain-free fabrics. For example, traditional superhydrophobic surfaces fail (i.e., become stained) when exposed to lowsurface-tension liquids, under pressure when impacted by a high-velocity stream of water (e.g., rain), and when exposed to physical forces such as abrasion and twisting. We have recently introduced slippery lubricant-infused porous surfaces (SLIPS), a self-healing, pressure-tolerant and omniphobic surface, to address these issues. Herein we present the rational design and optimization of nanostructured lubricant-infused fabrics and demonstrate markedly improved performance over traditional superhydrophobic textile treatments: SLIPS-functionalized cotton and polyester fabrics exhibit decreased contact angle hysteresis and sliding angles, omnirepellent properties against various fluids including polar and nonpolar liquids, pressure tolerance and mechanical robustness, all of which are not readily achievable with the state-ofthe-art superhydrophobic coatings.
\end{abstract}




\section{Introduction}

One of the major shortcomings in today's fabrics is obvious to identify, but difficult to overcome: they are easily stained, usually irreversibly, by a myriad of everyday substances due to the dirty, wet, and generally harsh environments, to which fabrics are subjected. Though the technology is still improving, the current state-of-the-art in repellent, superhydrophobic textiles wet and become stained when they interact with low-surface-tension liquids, biological fluids, hot liquids, complex or contaminated fluids, and/or when a liquid impacts with a high dynamic pressure. Enhancing the long-term stability and fundamental construction of current superhydrophobic textile endeavors will improve the quality of consumer clothing, tactical suits for military, medical gowns and lab coats, specialty garments for construction and manufacturing, agriculture, sports gear, shoes, etc. Herein we address these shortcomings by introducing omniphobic, pressure-stable and robust lubricant-infused nanocoatings [1-2] for offthe-shelf cotton and polyester fabrics.

Traditional superhydrophobic (TSH) surfaces are inspired by the lotus leaf [3]. These surfaces exhibit hydrophobic micro-/nano-scale roughness that creates a composite solid/air layer, which causes liquid droplets to bead on the tips of the nanostructures [4-5]. Highlights of current superhydrophobic fabric treatments include the in-situ growth of silica nanowires and nanoparticles, the grafting of polydimethylsiloxane or polyacrylic acid, and the direct incorporation of fluorinated compounds [6-14]. The resulting TSH materials are capable of repelling most aqueous solutions and are somewhat resilient to accumulation of dirt and small particles [15-19]. However, the practicality of this approach is hindered by the above inherent physical limitations of the lotus-effect approach $[1,6,19-20]$ : superhydrophobicity only exists in these materials when the air pockets are stable, but low-surface-tension fluids (or pressurized high-surface-tension fluids) can displace these air pockets and irreversibly penetrate the micro/nano-structured surface [21]. Further, damaged or infiltrated regions can serve as nucleation sites for ice formation or bacterial growth [22-24].

Lubricant-infused nano-/micro-structured surfaces have been recently introduced as an alternative approach to omniphobic materials [1-2,22-23,25-30]. The key feature of this design is the anchoring of a lubricating liquid into a chemically similar, texturized solid substrate, thus creating a stable, smooth, defect-free and liquid-repellent interface. While a TSH surface is dependent on the maintenance of the easily destabilized air pockets, such slippery lubricant- 
infused porous surfaces (SLIPS) maintain a robust interface between the lubricating film and the immiscible foreign liquid. Low-surface-tension liquids under pressure can penetrate the air pockets of lotus-inspired materials but they will not displace the stable lubricant interface. SLIPS can also be made optically transparent, self-healing, and tolerant to drastic changes in pressure, temperature, and $\mathrm{pH}[1,23,28-30]$. A suitable substrate for SLIPS must have micro-/nanoscale porosity with a strong chemical affinity for the lubricating film to ensure a complete wetting of the texturized solid by the lubricant and its stable retention in the porous network; surface area is crucial for increased capillarity and promotion of lubricant wicking, and chemical affinity provides strong adhesion to lock the lubricant onto the surface and prevent foreign materials from displacing the lubricant. While many techniques exist to confer surface roughness on various substrates, [14,31-34] we investigated the possibility of directly converting commercially available textile-based substrates into stable, lubricant-infused, omni-repellent fabrics.

We developed two methods to create the necessary nanoscale surface roughness: I) coating the textile fibers with silica micro-particles (SiM), and II) boehmite nanostructure formation on the textile fibers from sol-gel alumina treatment $(\mathrm{SgB})[7,10,14,23,28,35]$ (Figure 1). After nanostructure has been produced, it is necessary to fluorinate the substrate to facilitate chemical affinity of the surface with the perfluoro-polyether based lubricant. Hence, upon fluorination and subsequent infiltration with the lubricant, SLIPS-fabric can be produced from either approach. We applied the two surface modification methods to seven different types of fabric samples - two cotton and five polyester (PE) - and evaluated the non-wetting performance by quantifying static contact angle, contact angle hysteresis, liquid repellency after mechanical stress, pressure tolerance, and breathability. The characterization herein provides strong evidence that SLIPS-fabrics exhibit unique combination of liquid-repellency, durability, and pressuretolerance that are difficult to achieve based on state-of-the-art TSH materials.

\section{Materials and Methods}

\section{Sourcing fabrics}

The Dense polyester was purchased from Sew-Lew Fabrics, Cambridge, MA, the microfiber polyester was purchased from MicroFibres, Inc. and the Nike polyester was cut from Nike Dri-Fit 100\% polyester running shorts purchased from City Sports, Cambridge, MA. The rest of the fabrics were purchased from nearby fabric stores, including Sew-Lew in Cambridge, 
MA and Winmill Fabrics in Boston, MA. With regard to terminology, "fibers" are twisted together to make "threads", which are in turn woven to make the fabric.

\section{Surface activation of polyester fabrics}

This process is only required to prepare polyester fabrics for silica micro-bead deposition. Amines readily react with polyester by nucleophillic acyl cleavage of the ester linkages for surface activation [36]. Five to eight $2 \times 2 \mathrm{~cm}$ squares of polyester were first cleaned with DI water, ethanol, and then hexane. Fabrics were dried for at least $1 \mathrm{~h}$ at $70^{\circ} \mathrm{C}$ and further dried with a heat gun before adding to a $1 \%$ solution of aminopropyltriethoxysilane (APTES, Sigma Aldrich) in anhydrous toluene (Sigma Aldrich) and stirring for $24 \mathrm{~h}$ at $65^{\circ} \mathrm{C}$ under dry nitrogen. Samples were then removed, rinsed with toluene several times, and dried under vacuum. Dried samples were submerged in deionized water overnight, removed, rinsed with water, and dried for at least $3 \mathrm{~h}$ under vacuum before immersing in a $1 \%$ tetraethyl orthosilicate (TEOS) solution in water for 4-8 h. Samples were rinsed with water and dried overnight before silica particle deposition.

\section{Silica micro-particle synthesis/deposition}

In-situ polymerization of silica-microparticles onto cotton or activated polyester was performed using standard methods previously reported [7,10,14,35] to obtain a roughened substrate for SLIPS. Jersey cotton and Muslin were cleaned with water, ethanol, and isopropyl alcohol prior to reaction. The prepared samples were submerged into a 1:3 mixture of methanol and isopropanol, $20 \mathrm{~mL}$ ammonium hydroxide (Sigma Aldrich, St. Louis MO), and $12 \mathrm{~mL}$ TEOS (Sigma Aldrich, St. Louis MO) [7,35]. All solvents and chemicals were used without further modification. The mixture was stirred for $6 \mathrm{~h}$ at room temperature, and the samples were isolated and rinsed extensively with toluene several times. Dried fabrics were blown with compressed air to remove any residual detached particles that were not firmly attached to the fabric fibers. Subsequent fluorosilanization renders the fabric surface superhydrophobic.

\section{Fluorosilanization of silica micro-particle samples}

The roughened silica-bead surface was fluorosilanized either with $1 \mathrm{H}, 1 \mathrm{H}, 2 \mathrm{H}, 2 \mathrm{H}$ perfluorooctyltriethoxysilane (Sigma-Aldrich) or perfluorododecyl-1H,1H,2H,2H-triethoxysilane (Gelest). A solution of $4.8 \%$ silane stock and $>99.7 \%$ acetic acid were mixed in equal parts in 200 proof ethanol (i.e., in a 1:1:19 ratio of the above ingredients). After this, mixture was stirred 
for $60 \mathrm{~min}$ (to allow sufficient oligomerization), the fabrics were dipped into the mixture for 2-4 min and allowed to hang dry. The silane chains attach to the surface of the silica coating of the fabric and render the rough surface superhydrophobic [9,11,37]. Silica-microparticle (SiM) deposition is an effective method used to confer microscale surface roughness on cotton fabrics $[3,29]$. Figure 1 summarizes the steps required for this scalable process. Fewer steps are required to achieve the desired surface treatment for chemically reactive, hydroxyl-rich cotton fabrics, however further processing was necessary to induce covalent adherence of silica particles to more inert polyesters. Hence, we employed a two-step surface activation process whereby polyester cleavage using (3-aminopropyl)triethoxysilane (APTES) and subsequent reaction with TEOS created silica-like surface functionalities [35]. This process was optimized for PE as seen in SFigure 1. Chemical composition of each fabric surface was first confirmed in SFigure 2. Once silica-like surface chemistry was achieved, uniform particles were synthesized within all fabric samples to ultimately form a rough, nanostructured surface. Lastly, fabrics were dipped into an perfluoroalkyl-silane/ethanol solution to render the rough surface superhydrophobic, thus completing the SiM functionalization.

\section{Sol-gel boehmite treatment}

All cotton and polyester samples were oxygen plasma cleaned for $300 \mathrm{~s}$ (250 watts, oxygen flow of $15 \mathrm{~cm}^{3} / \mathrm{min}$ ). Cleaned samples were dipped in alumina sol-gel pre-cursor whose preparation procedure can be found in the supplementary information [18]. After $10 \mathrm{~min}$, the fabric was removed and dried overnight at $70^{\circ} \mathrm{C}$. Dried samples were immersed in a $95^{\circ} \mathrm{C}$ water bath for $15 \mathrm{~min}$ to create boehmite nanostructures, removed, dried, and then submerged in a $1 \%$ solution of FS-100, a perfluoroalkyl phosphate surfactant (Mason Chemical Company), in ethanol (Chemguard Inc., Mansfield, TX, USA) for $1 \mathrm{~h}$ at $70^{\circ} \mathrm{C}$. Samples were rinsed with ethanol and dried overnight before performing the contact angle and SEM analyses. Boehmite, formed in a reaction between aluminum and $80-100^{\circ} \mathrm{C}$ water, is a dense network of nano-scale $\mathrm{AlO}(\mathrm{OH})$ crossed leaflets that can be fluorinated to become an effective superhydrophobic surface. We used sol-gel approach schematically shown in Figure 1, to coat fabrics with boehmite nanostructures [18,31-34]. 


\section{Sample lubrication}

The surface of $\mathrm{SgB}$ or $\mathrm{SiM}$ functionalized samples has a strong affinity to fluorinated oils. To avoid excessive lubrication, we applied perfluoropolyether lubricant Krytox ${ }^{\mathrm{TM}}$ (Dupont Inc.) to wick through the sample and remove the excess by contacting the surface of the sample with a Kimwipe. About 30-100 $\mu \mathrm{L}$ of oil is needed to infuse $4 \mathrm{~cm}^{2}$ of the material, depending on the fabric thickness.

\section{Scanning electron microscopy (SEM) imaging}

SEM characterization was performed with a Zeiss Supra field emission microscope. Samples were coated by Pt-Pd sputtering for 60-150 s prior to SEM characterization.

\section{Contact angle analyses}

Contact angles were recoded using a contact angle goniometer (CAM 101, KSV Instruments, resolution $=0.01^{\circ}$ ) at room temperature. $10 \mu \mathrm{L}$ droplets of DI water were used for all static contact angle measurements. Contact angle hysteresis $(\mathrm{CAH})$ values were obtained by slowly increasing and decreasing droplet volume using a syringe needle while imaging the droplet movement, measuring advancing and receding contact angles, respectively, from these images, and subtracting the averages of these values. At least seven independent measurements were taken for static, advancing, and receding contact angles.

\section{Twisting test}

A $2 \times 3 \mathrm{~cm} \mathrm{SiM} \mathrm{or} \mathrm{SgB}$ fabric sample was secured between two medium sized clamp-type paper clips, and the assembly was hung by affixing one of the clips to a hook. By rotating the unbound lower clip, the fabrics were twisted $\pm 360^{\circ}$; the first twist was defined as a $360^{\circ}$ rotation clockwise followed by a return to rest position, the second twist was $360^{\circ}$ counterclockwise followed by a return to rest position, and so on. After the specified number of twists $(0,5$, or 50$)$, the sliding angle of a $20 \mu \mathrm{L}$ droplet of DI water was measured at least 3 times. The sliding angle is the tilting angle at which the droplet begins to slide along the surface without pinning. The sliding angle data and the SEM characterization provide a complete picture of the performance deterioration resulting from the twisting test.

\section{Rubbing test}

A $\mathrm{SgB}$ or SiM fabric sample was secured to a surface with tape and vigorously rubbed with a rolled up Kimwipe for approximately $10 \mathrm{~s}$. This is a preliminary abrasion test that 
simulates a contact with other fabrics or the surrounding environment. Damage was qualitatively observed by testing the repellency of water before and after rubbing, and SEM characterization showed the physical damage occurring to the nanostructure.

\section{Standardized repellency tests}

The American Association of Textile Chemists and Colorists (AATCC) test \#193 was used to analyze the repellency of non-lubricated (TSH) and lubricated (SLIPS) fabric samples to lowsurface-tension aqueous test liquids. This test is explained in detail in the AATCC technical manual [38]. Eight test liquids, composed of different volume fractions of IPA in de-ionized water, were prepared and numbered as shown in STable 1. Beginning with the first highest surface tension liquid, a test droplet was applied to the surface of the fabric sample and allowed to sit for $30 \mathrm{~s}$. The droplet was then observed to assess the wetting of the fabric: if the fabric is not wetted, then the process is repeated for the next test liquid, and if the surface is wetted then the fabric receives a score corresponding to the previously applied test liquid (i.e., the lowest surface tension liquid repelled by the fabric). If the test liquid only slightly wets the surface, the fabric is assigned a non-integer score halfway between the previous and current test liquid. A maximum score of 8 may be achieved, if the sample is not wetted by any of the test liquids.

The AATCC test \#188 was used to test repellency against alkanes of decreasing surface tension to characterize the repellency of oils and other nonpolar liquids [38]. This test is very similar to the aqueous liquid repellency test: the droplets were placed on TSH and SLIPS samples for $10 \mathrm{~s}$ before the wetting behavior was observed. The various hydrocarbon test liquids are listed in STable 2. Again, the lowest surface tension liquid that does not wet the surface of the liquid determines the score. Non-integer scores may be assigned, if only partial wetting occurs, and a maximum score of 8 is achieved when even test liquid 8 , the lowest surface tension liquid in the test, does not wet the surface of the fabric.

\section{Droplet impact test}

The tolerance of fabric samples to pressurized liquids of high and low surface tension was measured with the droplet impact test. A pipette was fixed $20.3 \pm 0.5 \mathrm{~cm}$ above a fabric sample immobilized on a tilting stage with double-sided tape. A $10 \mu \mathrm{L}$ test droplet was carefully ejected from the pipette and impacted the surface of the fabric at a controlled velocity, and the sliding angle of the droplet was measured immediately after impact. The dynamic pressure was 
estimated by $\mathrm{P}_{\text {dynamic }}=1 / 2 \rho \mathrm{V}^{2}$, where $\rho$ is the density of the liquid and $\mathrm{V}$ is the impacting velocity. The impact velocity was estimated using kinematic equations, and thus the tetradecane droplet exerts a dynamic pressure of $\sim 1520 \mathrm{~Pa}$ and the water droplet exerts a dynamic pressure of 1990 Pa. Irreversible pinning occurs for the superhydrophobic samples and cannot be recorded; the most important information comes from whether the droplet slides or does not slide.

\section{Breathability testing}

The breathability test was adapted from the standard ASTM E96-E upright cup water vapor transmission test. Each fabric sample was tested by a single 3D printed capsule; the inside of the capsule was dried by $20 \mathrm{~g}$ of Drierite desiccant (Drierite, Inc., Xenia OH) and separated from the moist air outside of the capsule by the fabric sample that was sealed onto the capsule by a ring-shaped cap clamped in place (SFigure 5). In between repeated experiments, the desiccant was regenerated by placing into a vacuum oven at $150^{\circ} \mathrm{C}$ overnight. The external environment of the chambers was carefully controlled in a custom made environmental chamber maintained at $50 \%$ relative humidity and $23 \pm 1^{\circ} \mathrm{C}$. Minimal airflow in the chamber prevented temperature gradients and inconsistencies. The water vapor was pulled into the chamber through the sample by the humidity gradient. After initial weighing, the test capsules were removed from the environmental chamber and weighed after 1, 2, 3, 4, 5, 6, 8, 22, $24 \mathrm{~h}$, and the mass increase of each chamber was plotted (SFigure 6 and 7). To confirm the omniphobicity, sliding angles of hexadecane and/or de-ionized water droplets on lubricated samples were recorded. The mass of the lubricated membranes (and thus the mass of the lubricant) before and after $24 \mathrm{~h}$ was also recorded. A typical experiment included up to 9 capsules running simultaneously. In each experiment, two controls were always present to ensure consistency in conditions between experiments: an open chamber without a membrane and a chamber sealed by Parafilm, which is impermeable to water vapor (Pechiney Plastic Company, Chicago, IL). Lubricated and untreated samples were tested against each other to observe the effect of SLIPS on the breathability of the fabric. Each sample was tested a minimum of three times, either with three separate samples in a single experiment or with one sample across three separate experiments.

\section{Results and Discussion}

Fabrics introduce unique physical features (hierarchical feature sizes coming from fiberthread-weave length scales), logistical considerations (cost, complexity of procedure), and 
demanding applications (requiring durability, breathability, etc.) into the design space of the final material. We chose two very common types of fabric: cotton and polyester (PE). They are inexpensive, readily available, widely used, and environmentally friendly [39]. The weave of the fabric is an important parameter since it inherently has a much more complex topography than a simple, flat surface. We investigated textiles that exhibited a range of thread sizes, weave densities, and weave patterns (Table 2). As discussed below, we observe some strong correlations between these parameters and liquid-repellent, anti-staining performance of SLIPSmodified fabrics.

To investigate the role of weave density in the development of effective omniphobic SLIPS-fabrics, we chose four square-weave fabrics with varying weave density: Dense PE, Nike PE, Crepe PE, and Muslin Cotton. Note that the weave density of the polyester fabrics ranges from a maximum of 400 threads $/ \mathrm{cm}^{2}$ (tightly woven Dense PE) to a minimum of 100 threads $/ \mathrm{cm}^{2}$ (loosely woven Crepe PE). We also tested three fabrics of different weave patterns, including the randomly oriented microfiber ( $\mu$ fiber) threads, the V-shaped weave of Gavadeen PE (Gav), and the column-based weave of the Jersey Cotton (J. Cotton) (Figure 2).

$\mathrm{SiM}$ and $\mathrm{SgB}$ treatment and surface fluorination according to the procedure outlined in Figure 1, resulted in fabrics uniformly covered with silica micro-particles $(\sim 150-500 \mathrm{~nm}$ in diameter) or boehmite nanoflakes, respectively (Figure 3). Droplets bounce off the surface of these fabrics (demonstrated in SMovie 1) and we observe static contact angles characteristic of superhydrophobic surfaces $\left(>150^{\circ}\right)$ (Figure $\left.4 \mathrm{~A}\right)$. SiM- and $\mathrm{SgB}$-treated fabrics were then infused with a perfluoropolyether lubricant (Krytox ${ }^{\mathrm{TM}}$, DuPont) that remains stably anchored in the textured substrate. These SLIPS-fabrics show an unprecedented ability to repel a wide range of fluids and to be resistant to staining (SMovie 2 and 3). Note that the presence of the Krytox lubricant on the fabric surface slightly affects the hand feel of the fabric - while the fabric does not feel wet, it may leave behind a slippery residue when touched if the lubricant is applied in excess. The residue can be further avoided by removing the excess lubricant that is not affixed to the surface with the conditioning step used in this investigation (i.e., blotting the surface with a Kimwipe)

To determine the optimal SLIPS fabric parameters, we investigated the static contact angle, contact angle hysteresis, omniphobicity, pressure resistance, durability, and breathability. We conducted three phases of testing on a successively smaller set of samples. See Table 1 for a summary of the fabrics used in each phase of testing. 


\section{Phase I: Hydrophobic and Slippery Behavior}

To begin Phase I characterization, we measured the static contact angle on all fabrics to quantify the hydrophobicity of non-lubricated (TSH) and lubricated (SLIPS) samples (Figure 4A). Each non-lubricated sample has a static contact angle in the range of $150-160^{\circ}$, and when the Krytox lubricating film is applied this angle decreases to approximately $110-120^{\circ}$. To quantify the repellency of the fabrics, we measured the contact angle hysteresis (CAH), which is the difference between advancing and receding contact angles as a droplet slides on a surface and directly relates to droplet mobility on a surface. We observe low CAH on almost all SLIPSfabric samples. Figure 4B shows all CAH data for the 14 fabric samples (see STable 3 for numerical values plotted in Figure 4). In the case of non-lubricated fabrics, there are multiple sources of pinning, including fibrillar protrusions, structural defects, and perhaps incomplete fluorination leaving hydrophilic areas on the surface. $\mathrm{CAH}$ values increase with increasing density of defects, or pinning points, on the surface of the material. Application of a lubricant dramatically reduces hysteresis for every fabric sample except for J. Cotton and Crepe PE droplets easily slide over the smooth surface created by the lubricating film.

Fluorinated surface functionality on the substrate is essential to maintain the integrity of the lubricant layer. The ideal SLIPS comprises of a polytetrafluoroethylene base infiltrated with perfluoropolyether lubricants (Krytox). The matching fluorinated surface chemistry and the highly fluorinated lubricant provides a strong enough interaction to affix the lubricant to the substrate. To demonstrate the necessity of fluorosilanization of SLIPS substrates, contact angle hysteresis for non-fluorinated, lubricated Dense PE was compared to fluorinated, lubricated Dense PE. Fluorosilanization, enabled by APTES activation, caused the contact angle hysteresis to decrease from $22.77^{\circ} \pm 8.76$, to $12.67^{\circ} \pm 5.13$. Non-lubricated controls exhibit contact angle hysteresis of $23.40^{\circ} \pm 10.15$, a value that is not significantly different from the lubricated but non-fluorinated samples. This clearly shows that lubricant is displaced from the non-fluorinated sample and fluorosilanization is required to maintain an immobilized lubricant layer.

In the case of Gavadeen PE treated with SiM-SLIPS, which has a static contact angle of $156.6^{\circ} \pm 3.1$ and a hysteresis value of $5.35^{\circ} \pm 3.1$, we see a combination of superhydrophobic and SLIPS-type performance. The lubricant entrapped within and around each nanostructured thread prevents pinning even if the test liquid is partially exposed to the non-lubricated, superhydrophobic surface, a scenario suggested by a relatively high static contact angle and a 
relatively low hysteresis value. Thus, we have engineered fabrics that combine slippery performance with both SLIPS and TSH attributes for excellent overall water repellency.

\section{Phase II: Robustness}

Reducing the sample pool with the selection criteria discussed earlier (Table 1), we tested which treatment method - $\mathrm{SgB}$ or $\mathrm{SiM}$ - is more robust when subjected to rubbing and twisting, as observed by the effect of twisting on sliding angle and coating integrity as studied by SEM. These experiments simulate the expected wear that fabrics may experience in most functional applications.

The twist testing data are shown in Figure 5. We twisted the fabric samples $+/-360^{\circ}$ with a custom-made setup (SMovie 4), and tested the sliding angle of water (20 $\mu \mathrm{L}$ droplet volume) on a fabric sample lubricated with Krytox 102 after 0, 5, and 50 twists. Sliding angles that exceeded $35^{\circ}$ were not measured because of experimental constraints and the large variability associated with strong pinning behaviors. Note that the red colored test water droplet did not wet any of the fabric samples after twisting 50 times. Remarkably, even when pinning was observed, the colored test water droplets could be rinsed away without leaving a stain.

$\mathrm{SgB} \mathrm{Gav}$. and $\mathrm{SgB}$ M. Cotton were the worst performers in the twisting test: both fabrics failed to slide at $35^{\circ}$ even before twisting, and we qualitatively observed droplet pinning to worsen with further twisting. For those samples whose sliding angles remain less than $35^{\circ}$, we can see a clear difference emerging between the $\mathrm{SgB}$ samples and the SiM samples: for the $\mathrm{SgB}$ treated samples, there is a significant increase in the sliding angle for 0, 5, and 50 twists, while on the SiM treated samples there is either no significant increase, or an initial increase that stabilizes with additional twisting. The most telling result comes from comparing SgB Nike PE with the SiM variant: the $\mathrm{SgB}$ sample shows a clear, almost linear increase in the sliding angle with increased number of twists, while the SiM sample shows no significant change.

An increase in sliding angle indicates that damaged nanostructures give rise to a decreased affinity of the lubricant to the fiber surface either due to the loss of nanostructure or due to cracks exposing surfaces that are not fluorinated. SiM fabrics exhibit more durable nanostructures than SgB fabrics. SEM images of the Nike PE fabric treated with both $\mathrm{SgB}$ and SiM, before and after twisting, confirm this (Figure 3). The SiM layer on the Nike PE fabric showed only minimal damage after 50 twists, whereas the boehmite shows smoothening and flattening of its vital nanostructures. Self-healing behavior arises in SLIPS from a redistribution 
of the lubricant to cover moderate damage and to continue to provide omniphobicity. In this way, the liquid-repellent performance of SLIPS-fabrics is less susceptible to damage than that of TSH fabrics. The extensive damage of the $\mathrm{SgB}$ fabrics diminishes capillarity and therefore the lubricant's ability to redistribute. This effect is not seen on the more durable SiM treatment. SiM Nike PE maintained the same sliding angle throughout twisting and $\mathrm{SgB}$ Nike PE experienced a continuous increase in sliding angle as the nanostructures became critically damaged. Therefore, with respect to robustness, lubricated SiM-treated fabrics show best performance.

For additional durability characterization, we vigorously rubbed non-lubricated fabric samples with a Kimwipe, qualitatively observed the repellency (SMovie 5 and 6), and characterized the surface with SEM (Figure 3). Though the ability to repel water appears to remain unaffected, SEM characterization reveals cracking damage on the $\mathrm{SgB}$-treated fabrics and no damage to the SiM-treated fabric (Figure 3). Specifically, rubbing causes the alumina shell to crack and detach from the fiber surface, in a fashion similar to the twisting test. The adhesion of the sol-gel alumina to the fibers was not fully optimized yet to provide sufficiently strong damage tolerance against rubbing. In contrast, the silica microparticles that are covalently attached to the fabric surface show strong adherence between the silica shell and the fiber. Therefore SiM-treated SLIPS-fabrics maintain omniphobic performance even when subjected to abrasion. We also observe that washing machine cycles have little effect on the integrity of the nanostructures (STable 4). We reiterate that damage to the nanostructures leads to premature loss of the lubricant and creation of new pinning points, drastically reducing the functional lifetime of the fabric.

\section{Phase III: Omniphobicity, pressure tolerance and breathability}

Given the results of the twisting and rubbing tests described above, we selected the SiMtreated fabrics for Phase III testing. We chose M. Cotton, Dense PE, Nike PE, and $\mu$ fiber to complete the characterization of the SLIPS fabrics and show the best overall performance. We performed water and hydrocarbon resistance testing to observe the repellency of low-surfacetension fluids, drop impact testing to determine the pressure tolerance of the fabrics, and water vapor transmission testing to characterize the fabric's breathability.

For each of the Phase III fabrics, a SLIPS (lubricated) sample was tested against a nonlubricated sample that serves as a representative TSH control. Liquid droplets of progressively lower surface tension (ranging from $72 \mathrm{mN} / \mathrm{m}$ for pure water to $24.0 \mathrm{mN} / \mathrm{m}$ for $60 \%$ isopropyl 
alcohol) were applied to fabric samples until the test droplet wets the surface (SFigure 3). The scores for the four samples are shown in Table 3. Clearly, the lubricated, SLIPS-fabric samples exhibit a higher score than their non-lubricated, superhydrophobic counterparts. In other words, the presence of the thin lubricating film around the threads prevents penetration of low-surfacetension liquids that would have otherwise wet the non-lubricated fabric. The Dense PE achieved the maximum score of 8: 60\% IPA in water did not wet the sample and could slide off without pinning. M. cotton, Nike PE, and $\mu$ fiber PE were capable of repelling aqueous liquids down to surface tensions of $26.5,25.0$, and $24.5 \mathrm{mN} / \mathrm{m}$, respectively. A particularly interesting trend emerges from these results: the scores for the SLIPS-fabric samples correlate with increasingly tight weaves. M. Cotton has the loosest weave and experiences the most pinning; Dense PE has the tightest weave and thus performs the best. We attribute this trend to the overall smoothness of the SLIPS-fabric surface where even sub-millimeter scale roughness can still slightly compromise the ultrasmooth nature of lubricant-infused interface [28].

To extend the testing to organic liquids, we tested the repellency of the Phase III fabrics against mineral oils and alkanes of progressively shorter chain length and lower surface tension (SFigure 4, STable 2). Table 4 summarizes the hydrocarbon repellency scores for the Phase III fabric samples. All test organic droplets pinned to the TSH fabrics and easily slid off of the lubricated, SLIPS-fabric samples. The TSH samples, particularly the M. Cotton and Dense PE, generally received lower scores than in the aqueous repellency test, indicating that organic liquids with even lower surface tensions are more prone to infiltrating the spaces within a fabric. Despite this, the scores of the lubricated samples in both the hydrocarbon and aqueous tests were within \pm 1 from each other and follow the same trend of larger weave patterns causing reduced repellency of low-surface-tension liquids. Again, the dense polyester sample showed repellency to all of the test liquids and achieved the highest possible score of 8 . SLIPS-fabric of a sufficiently dense weave can support a lubricating film that repels liquid compounds of broad compositions, polarities and surface tensions, which is a remarkable advancement to stainresistant, fabric-based materials.

Another important advantage of a lubricated fabric is that it maintains its slippery, omniphobic performance under pressure. To assess the pressure stability of the Phase III fabric samples we used the drop impact test as outlined in $[1,44]$. The results for the drop impact test are shown in Figure 6: for a liquid of a given surface tension, the sliding angle is determined 
immediately after the droplet impacts the surface with the shown dynamic pressure. The SiMSLIPS Nike PE and Dense PE fabrics retain their liquid repellency at high pressures (>1500 Pa) while typical lotus-type TSH surfaces fail at $400 \mathrm{~Pa}$ [45]. The sliding angle of the test liquids on SiM-SLIPS treated $\mu$ fiber fabric increases after high impact, however sliding is still observed. This indicates that there is a SLIPS layer penetrated by the many protruding fibers on its disordered surface. Sliding angles for non-lubricated samples are not included because this pressure is above the threshold at which the Cassie-to-Wenzel transition occurs; water droplets are strongly pinned to the surface and will not slide even from the vertical surfaces, while tetradecane droplets simply wet the fabric as expected. SMovie 7 dramatically demonstrates the effect we describe: SiM-SLIPS Nike PE shows sliding angles below $10^{\circ}$ (10 $\mu \mathrm{L}$ droplet) after a collisional pressure applied by the falling test liquid, while un-lubricated SiM Nike PE shows irreversible pinning in the same conditions. Figure 6 shows that liquids of different surface tensions and dynamic impact pressures do not cause prominent increases in sliding angle of the SLIPS fabrics. As could be expected based on tightness of fabric weave and surface flatness, the Dense PE and Nike PE both show the best performance in this test with post-impact sliding angles of $8.8 \pm 1.0^{\circ}$ and $20.9 \pm 2.0^{\circ}$. The $\mu$ fiber showed an increase of $\sim 10^{\circ}$ in sliding angle after impact pressure due to the presence of loose fibers oriented approximately normal to the surface of the fabric. It is worth noting that despite droplet pinning the lubricated fabric was neither wet nor stained by the test liquid and the pinned droplets could be easily washed off the surface leaving no residue.

Breathability, or more specifically, water vapor transmission rate (WVTR), is an important factor in determining suitable applications for SLIPS fabrics. For each experiment, we tested a non-lubricated and lubricated sample alongside two controls: a capsule sealed by (impermeable) Parafilm and an open capsule (SFigure 5). Table 5 summarizes the WVTR mass change after $24 \mathrm{~h}$ for the fabrics and PTFE controls. All of the SLIPS samples (lubricated with Krytox 102) except for M. Cotton did not show a statistically significant difference in breathability from that of the Parafilm control (statistical tests shown in STable 5 and SFigure 6). Non-lubricated $\mu$ fiber, Nike PE, and Cotton samples exhibit similar breathability despite large differences in their relative weave pattern and weave density. Also, the $\mu$ fiber, Nike PE, and Dense PE all show no breathability (i.e., no difference from the Parafilm control) while Cotton, the least densely woven fabric, shows significant (but still low) breathability. This substantiates 
the presence of a certain macro-scale pore size threshold above which the Krytox does not wick across, leaving a space through which air and water vapor can flow (see Supplementary for details).

\section{Conclusions}

While lotus-effect superhydrophobic surfaces have been thoroughly investigated for years and continue to show improvement, their design has some fundamental shortcomings that will always limit omniphobicity, stain resistance, durability and pressure tolerance. SLIPS overcome these problems, and we have engineered nanostructured coatings that achieve the promising benefits using readily available fabrics as a substrate. We have successfully introduced simple methods by which to create SLIPS nanocoatings on cotton and polyester fabrics. We characterized SLIPS-coated fabrics against superhydrophobic standards and showed that the lubricated structured surfaces display superior pressure-stable and damage-tolerant repellency to polar and non-polar liquids.

In practice, these lubricated nanostructure-coated fabrics will be able to repel water, oil, dirt and mud; therefore, tents, boots, and other outerwear would be significantly improved. In applications where the material may be touched frequently, the slippery residue can be mitigated with a thin protective layer that prevents direct contact, or perhaps with more conservative lubrication. Most importantly, however, the presented iteration of SLIPS fabrics may provide a unique solution as a stable, anti-fouling material for specialty suits in demanding applications in extreme, contaminated environments for which no solutions exist and where breathability is not the most critical factor. Such applications may include tactical suits for military, medical gowns and lab coats, and specialty garments for construction and manufacturing. Furthermore, antiicing properties would provide advantage in large awnings, tents and open-air buildings like sports stadiums. We believe that such SLIPS-fabric is a novel material that, for the first time, confers pressure- and damage-tolerant omniphobicity on fabric-based substrates. 
Table 1. Inventory of treated fabrics used for each phase of experiments.

\begin{tabular}{|c|c|c|c|c|c|c|c|}
\hline \multirow{2}{*}{ Fabric Name } & \multirow{2}{*}{ Abbreviation } & \multicolumn{2}{|c|}{ Phase I } & \multicolumn{2}{|c|}{ Phase II } & \multicolumn{2}{|c|}{ Phase III } \\
\hline & & SgB & $\mathrm{SiM}$ & SgB & SiM & SgB & SiM \\
\hline Muslin Cotton & M. Cotton & - & - & - & - & & • \\
\hline Jersey Cotton & J. Cotton & - & • & • & & & \\
\hline Dense Polyester & Dense PE & $\bullet$ & $\bullet$ & & $\bullet$ & & $\bullet$ \\
\hline Nike Polyester & Nike PE & - & - & - & - & & - \\
\hline Microfiber Polyester & $\mu$ fiber & $\bullet$ & $\bullet$ & & $\bullet$ & & $\bullet$ \\
\hline Gavadeen Polyester & Gav. & - & - & $\bullet$ & & & \\
\hline Crepe Polyester & Crepe & - & - & - & & & \\
\hline
\end{tabular}


Table 2. Specification of tested fabrics.

\begin{tabular}{|c|c|c|c|c|}
\hline Fabric & $\begin{array}{l}\text { Approximate } \\
\text { Thread Width }\end{array}$ & $\begin{array}{c}\text { Approximate } \\
\text { Threads per Inch }\end{array}$ & $\begin{array}{c}\text { Surface Weave } \\
\text { Pattern }\end{array}$ & Comments \\
\hline M. Cotton & $350 \mu \mathrm{m}$ & 32 & Square & $\begin{array}{l}\text { Largest thread; } \\
\text { least densely } \\
\text { woven }\end{array}$ \\
\hline J. Cotton & $200 \mu \mathrm{m}$ & 54 & V-shaped & $\begin{array}{l}\text { Many loose } \\
\text { threads }\end{array}$ \\
\hline Dense PE & $150 \mu \mathrm{m}$ & 69 & Square & $\begin{array}{l}\text { Smallest thread; } \\
\text { most tightly } \\
\text { woven }\end{array}$ \\
\hline Nike PE & $200 \mu \mathrm{m}$ & 55 & Square & Smooth surface \\
\hline$\mu$ fiber & $\begin{array}{c}\mathrm{N} / \mathrm{A}, \text { fiber width } 5 \\
\mu \mathrm{m}\end{array}$ & N/A & Random & $\begin{array}{c}\text { Disordered, } \\
\text { hairy structure }\end{array}$ \\
\hline Gav. & $300 \mu \mathrm{m}$ & 58 & V-shaped & $\begin{array}{l}\text { Diagonally } \\
\text { ridged surface }\end{array}$ \\
\hline Crepe & $300 \mu \mathrm{m}$ & 22 & Square & Loosely woven \\
\hline
\end{tabular}


Table 3. AATCC 193 aqueous liquid repellency test results.

\begin{tabular}{c|cc}
\multirow{2}{*}{ Sample } & \multicolumn{2}{|c}{ AATCC 193 Aqueous Liquid Repellency Score* } \\
\cline { 2 - 3 } & SiM Treatment - dry & $\begin{array}{c}\text { SiM Treatment - } \\
\text { lubricated }\end{array}$ \\
\hline M. Cotton & 5 & 5.5 \\
Dense PE & 5.5 & 8 \\
Nike PE & 4 & 6.5 \\
Hfiber & 5 & 7
\end{tabular}

*No standard deviations are shown because of the pass/fail nature of the test and the consistency of the results.

Table 4. AATCC 118 Hydrocarbon Resistance Test Results.

\begin{tabular}{c|cc}
\multirow{2}{*}{ Sample } & \multicolumn{2}{|c}{ AATCC 118 Hydrocarbon Resistance Score* } \\
\cline { 2 - 3 } & SiM Treatment - dry & $\begin{array}{c}\text { SiM Treatment - } \\
\text { lubricated }\end{array}$ \\
\hline M. Cotton & 2 & 5.5 \\
Dense PE & 3.5 & 8 \\
Nike PE & 5 & 7 \\
$\mu$ fiber & 4.5 & 6
\end{tabular}

*No standard deviations are shown because of the pass/fail nature of the test and the consistency of the results. 
Table 5. Summary of $24 \mathrm{~h}$ mass changes for all membranes*.

\begin{tabular}{|c|c|c|c|}
\hline Material & Lubrication & $\begin{array}{c}\text { WVTR } \\
\left(\mathrm{g} / 24 \mathrm{~h} / \mathrm{m}^{\wedge} 2\right)\end{array}$ & Fold Change \\
\hline No Membrane * & Control & $947.5 \pm 38.7$ & \multirow{3}{*}{25.5} \\
\hline & & & \\
\hline Parafilm * & Control & $37.2 \pm 18.4$ & \\
\hline$\mu$ fiber * & None & $497.5 \pm 61.4$ & \multirow{3}{*}{11.1} \\
\hline & & & \\
\hline$\mu$ fiber * & K102 & $44.6 \pm 6.8$ & \\
\hline Nike PE * & None & $507.5 \pm 70.9$ & \multirow{3}{*}{11.5} \\
\hline & & & \\
\hline Nike PE * & K102 & $44.2 \pm 15.6$ & \\
\hline Dense PE * & None & $270.5 \pm 36.6$ & \multirow{3}{*}{6.1} \\
\hline & & & \\
\hline Dense PE * & K102 & $44.0 \pm 22.4$ & \\
\hline M. Cotton * & None & $493.3 \pm 17.1$ & \multirow{3}{*}{3.5} \\
\hline & & & \\
\hline M. Cotton * & K102 & $139.4 \pm 17.9$ & \\
\hline 200 nm PTFE * & None & $473.9 \pm 35.8$ & \multirow{3}{*}{11.3} \\
\hline & & & \\
\hline 200 nm PTFE * & K102 & $42.0 \pm 8.0$ & \\
\hline $1 \mu \mathrm{m}$ PTFE * & None & $470.9 \pm 41.1$ & \multirow{3}{*}{14.9} \\
\hline & & & \\
\hline $1 \mu \mathrm{m}$ PTFE * & K102 & $31.6 \pm 18.7$ & \\
\hline $20 \mu \mathrm{m}$ PTFE * & None & $483.4 \pm 49.0$ & \multirow{3}{*}{14.1} \\
\hline & & & \\
\hline $20 \mu \mathrm{m}$ PTFE * & K102 & $34.2 \pm 19.6$ & \\
\hline Punc. 200 nm PTFE * & None & $480.5 \pm 39.6$ & \multirow{3}{*}{3.7} \\
\hline & & & \\
\hline Punc. 200 nm PTFE * & K102 & $131.1 \pm 76.0$ & \\
\hline
\end{tabular}

*Statistical comparisons (student's one-tailed t-test, significance level $\mathrm{p}<0.05$ ) were made between the lubricated and un-lubricated iterations of each fabric; in all pairs, the lubricated fabric showed a large decrease in breathability. There was no significant difference between the breathability of the lubricated fabrics/membranes and the Parafilm control, except in the case of Muslin cotton. See STable5 for p-values. 


\section{Figures}

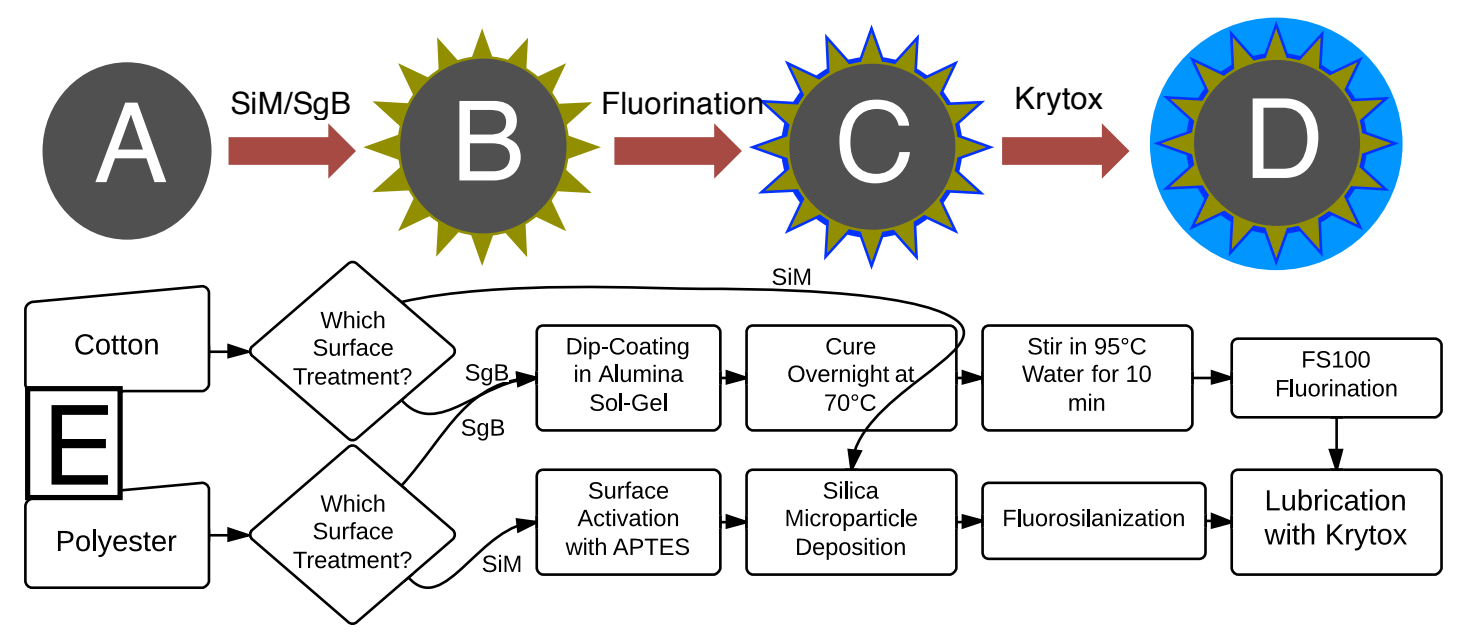

Figure 1. Design principles of lubricated nanostructured fabrics (SLIPS-fabrics). A single, bare fiber being functionalized with SLIPS is depicted in a schematic (A-D). A bare fiber (A) is roughened with the silica micro-particle $(\mathrm{SiM})$ or sol-gel boehmite $(\mathrm{SgB})$ approach $(\mathrm{B})$ and fluorinated to achieve chemical similarity to (perfluoroether)polymer Krytox (C) before the lubricating film is applied (D). This confers pressure-tolerant, self-healing repellency against a broad range of fluids. The flow chart (E) contains more specific information regarding the SiM and $\mathrm{SgB}$ functionalization protocols applied to cotton and polyester fabrics. 


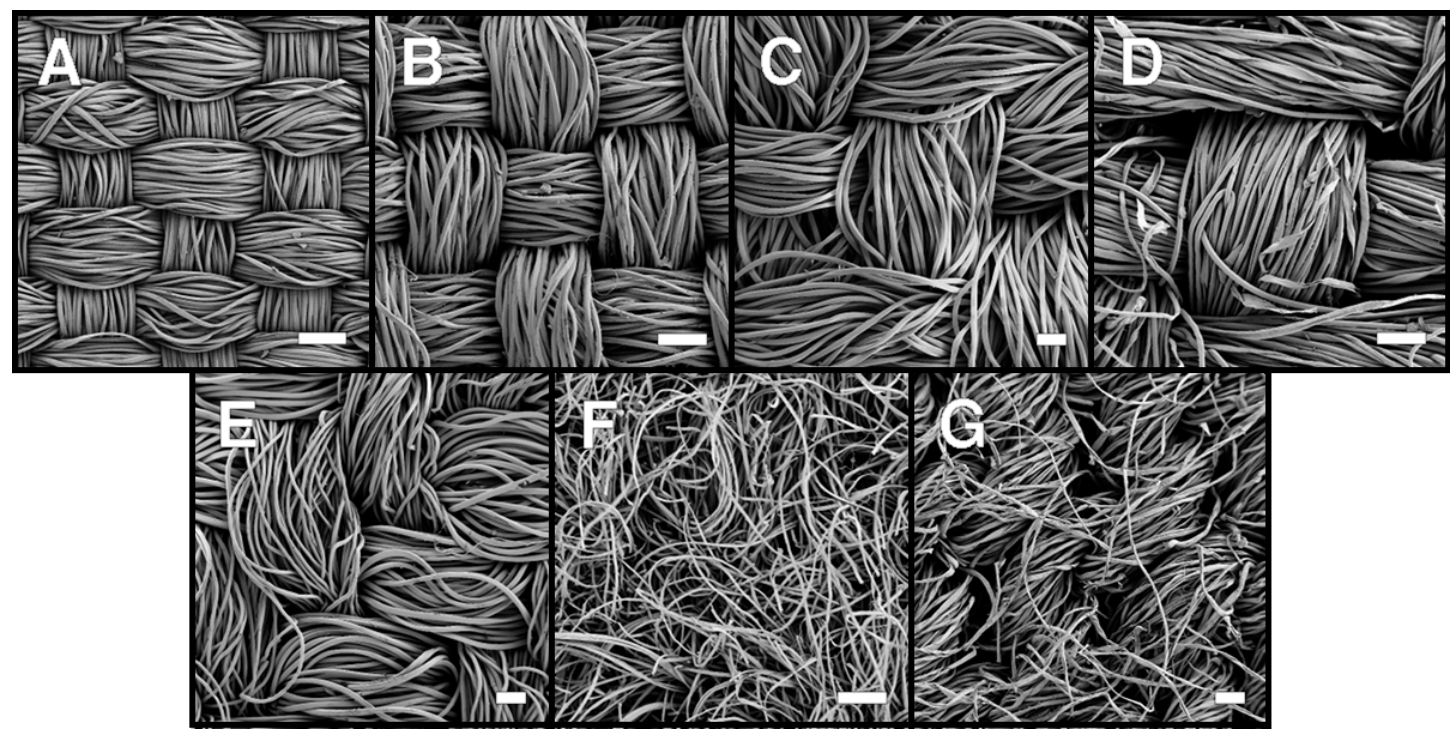

Figure 2. SEM Images of the weave pattern of each fabric. Square-weave fabrics are arranged along the top row (A-D) in order of decreasing weave density, and the fabrics of other weaves are arranged along the bottom row (E-G). The two cotton fabrics are on the right edge of the figure $(D, G)$. For further description of these fabrics please refer to Table 2. The fabrics shown are Dense PE (A), Nike PE (B), Crepe PE (C), Muslin Cotton (D), Gavadeen PE (E), $\mu$ fiber (F), and Jersey Cotton $(\mathrm{G})$. Scale bars are $100 \mu \mathrm{m}$. 


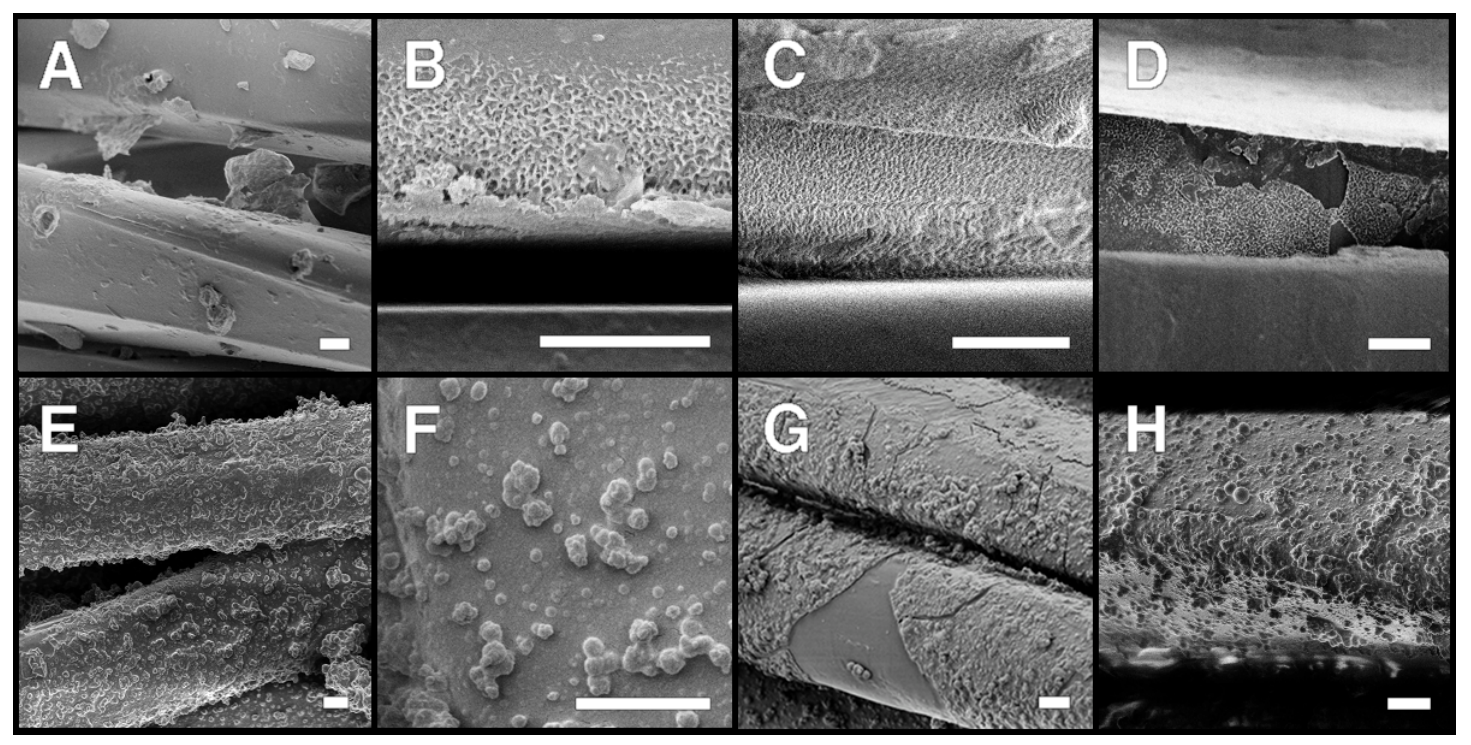

Figure 3. SEM characterization of fabric treatments. A scanning electron microscope was used to evaluate the surface roughness and durability of the sol-gel boehmite (SgB) (A-D) and silica microparticle $(\mathrm{SiM})(\mathrm{E}-\mathrm{H})$ treatments on Nike polyester fabric. All scale bars are $2 \mu \mathrm{m}$. Freshly treated $\mathrm{SgB}$ fibers (A) show full coverage of the fiber with $\mathrm{SgB}$; dramatic microbead coverage is apparent on freshly treated SiM fibers (E). High magnification of the microstructures $(\mathrm{B}, \mathrm{F})$ reveals the surface roughness that facilitates good SLIPS performance. When twisted 50 times, smoother boehmite is still present $(\mathrm{C})$ in crevices between fibers, while the outside of the fibers have become smooth. Also after 50 twists, the SiM threads (G) exhibit some cracking while maintaining good microparticle coverage. After vigorous rubbing with a Kimwipe, $\mathrm{SgB}$ fabric (D) exhibits cracking and smoothness on the outer fibers, while under the same conditions the SiM coating remains intact $(\mathrm{H})$. 

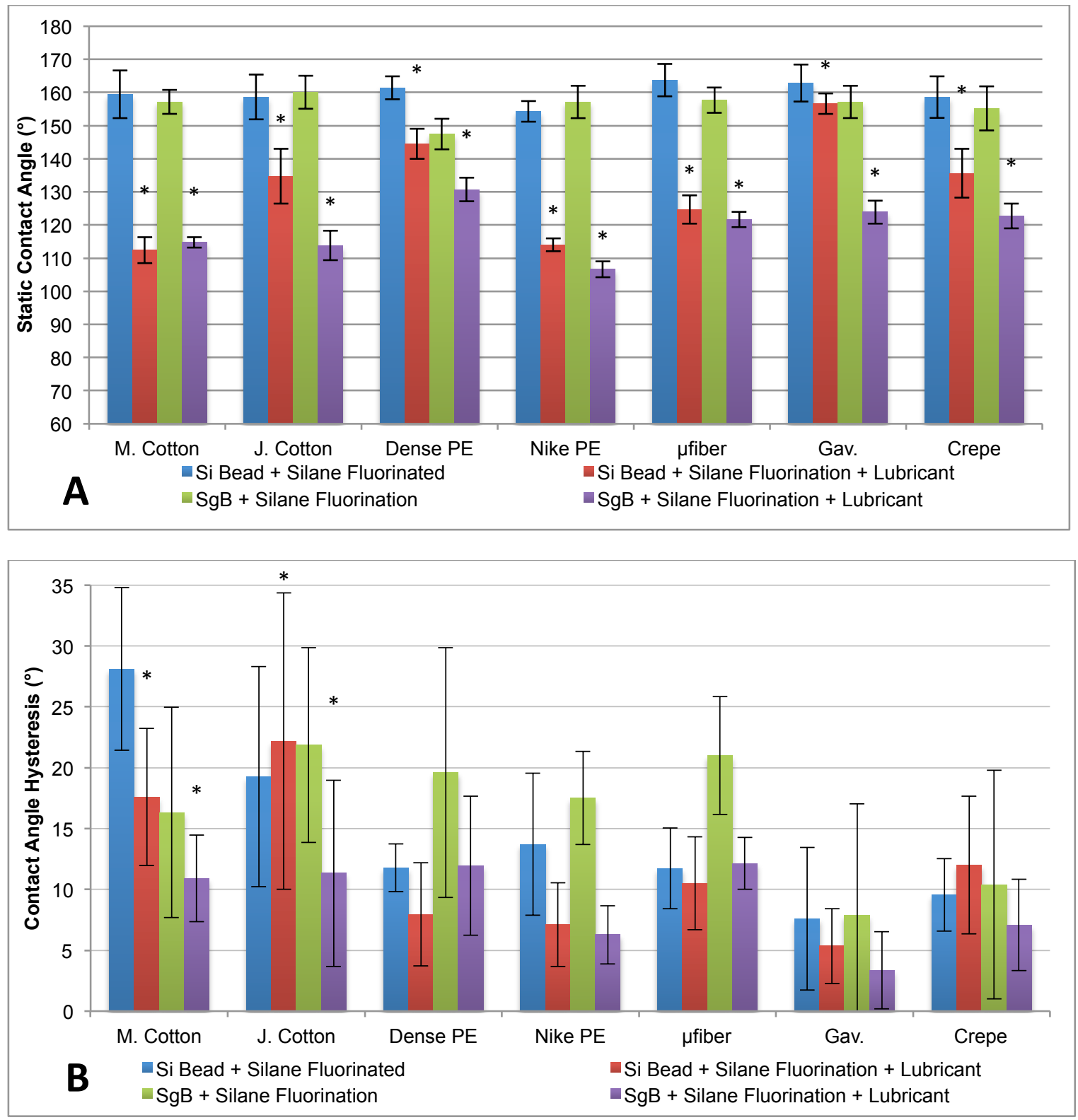

Figure 4. Static contact angles (A) and contact angle hysteresis data (B) for all functionalized fabrics. Fabric samples, both un-lubricated and lubricated with Krytox 102 (K102), were functionalized with either silica microbeads ( $\mathrm{SiM})$ or sol-gel boehmite $(\mathrm{SgB})$. Contact angles were measured using a contact angle goniometer. (A) A $10 \mu \mathrm{L}$ water droplet was placed onto the surface of the fabric sample for measurement. (B) The advancing and receding contact angles were recorded and these values were subtracted to determine the hysteresis. $N=7$; error bars are +/- SD. Asterisks denote statistically significant results (Student's two-tailed t-test, $\mathrm{P}<0.05$ ); comparisons are only made between $\mathrm{SiM}+\mathrm{K} 102$ and $\mathrm{SgB}+\mathrm{K} 102$ for each fabric sample. 


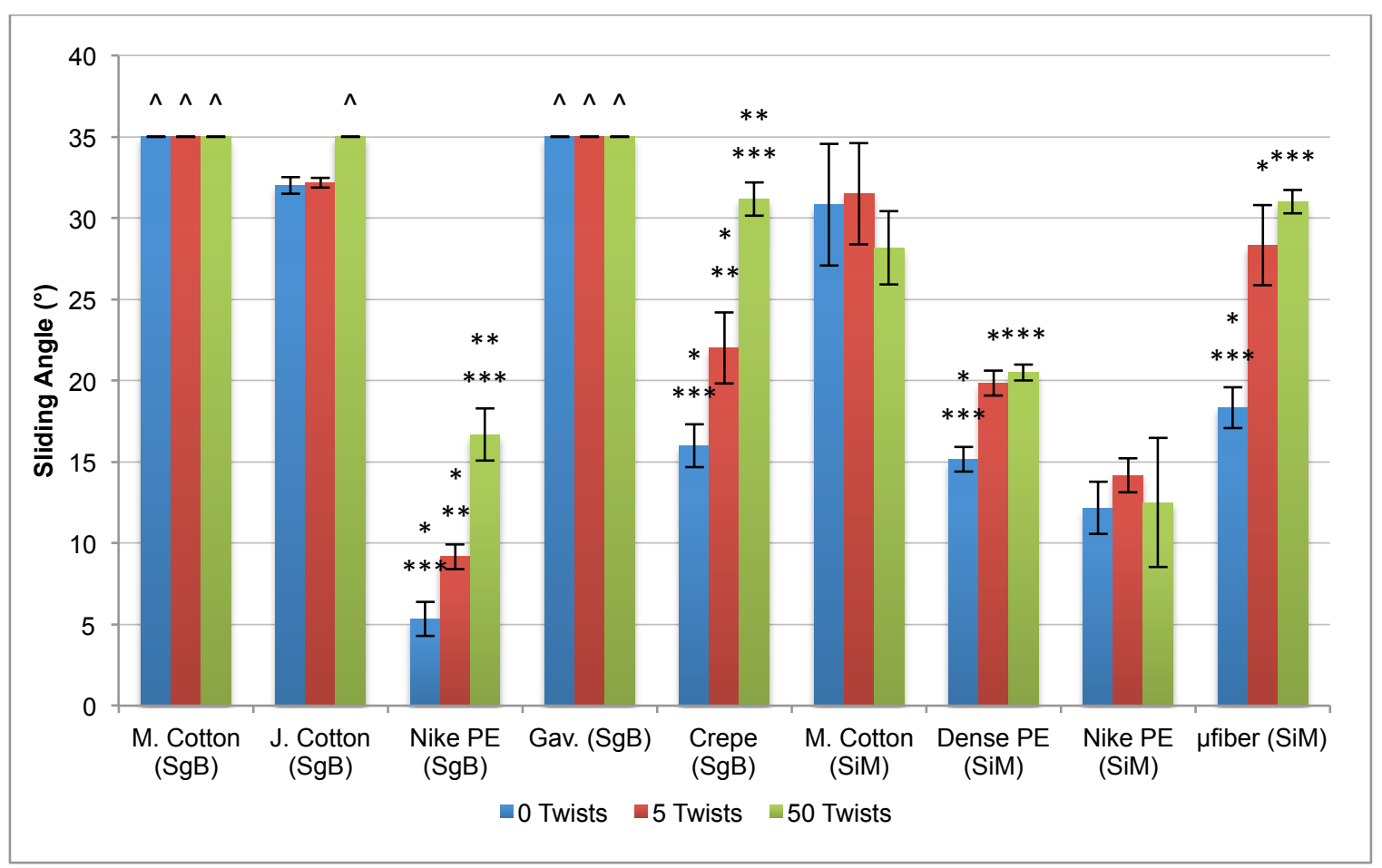

Figure 5. Twisting test results (robustness characterization). The sliding angle for a $20 \mu \mathrm{L}$ water droplet on $\mathrm{SgB}$ and SiM SLIPS-fabrics after twisting for $\pm 360^{\circ} 0,5$, and 50 times was subsequently measured. Some samples exhibited strong pinning, and no measurements were taken for sliding angle $>35^{\circ}$. $\mathrm{N}=3$; error bars are $+/-$ SD. Asterisks show statistical significance (Student's two-tailed t-test, $\mathrm{P}<0.05)$. Arrows $(\wedge)$ represent measurements that were above $35^{\circ}$. 


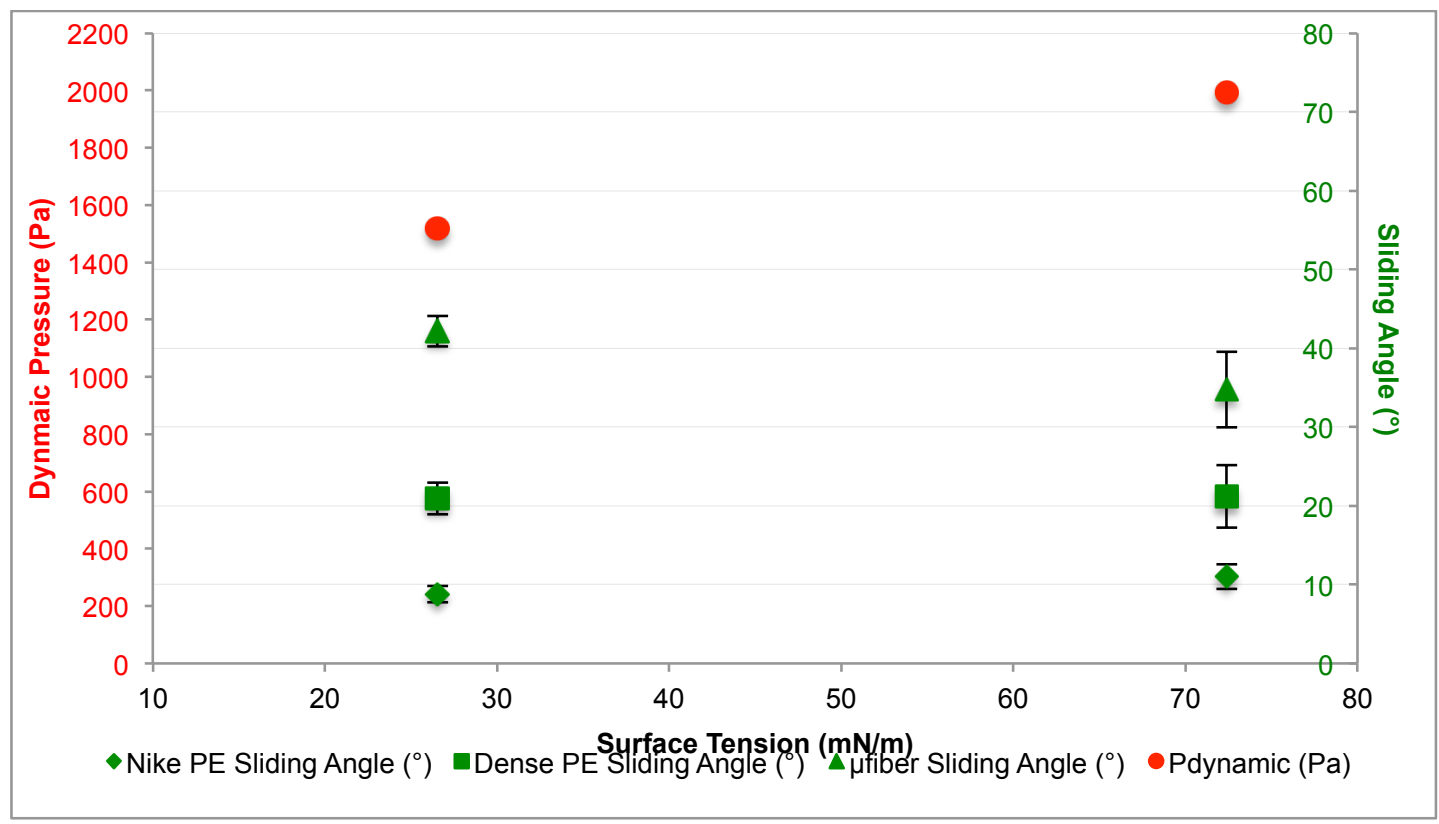

Figure 6. Drop impact characterization of SLIPS-treated fabrics. Water (surface tension = $72.4 \mathrm{mN} / \mathrm{m}$ ) and Tetradecane (surface tension $=26.55 \mathrm{mN} / \mathrm{m}$ [45]) were dropped from a height of $20.4 \mathrm{~cm}$ to achieve a dynamic pressure shown by the red circle markers. The $10 \mu \mathrm{L}$ droplet impacted Nike PE, Dense PE, and $\mu$ fiber lubricated fabrics treated with SiM, and the sliding angle was subsequently measured.

\section{Acknowledgement}

We thank Tom Blough and Jack Alvarenga for their help with the experimental setup. The work was supported partially by the Advanced Research Projects Agency-Energy (ARPA-E), U.S. Department of Energy, under Award Number DE-AR0000326. We acknowledge the use of the facilities at the Harvard Center for Nanoscale Systems supported by the NSF under award ECS0335765.

\section{Abbreviations}

SiM, Silica-Microparticle; SgB, Sol-gel boehmite; SLIPS, slippery liquid-infused porous surfaces

\section{References}

[1] Wong T S et al 2010 Nature 477443

[2] Lafuma A and Quere D 2011 Europhysics Letter 9656001

[3] Barthlott W and Neinhuis C 1997 Planta 2021

[4] Nosonovsky N and Bhushan B 2009 Curr. Opin. Colloid Interface Sci. 14270

[5] Bormashenko E 2010 Philos. Trans. R. Soc. London, Ser. A 3684695 
[6] Quere D 2005 Reports on Progress in Physics 682495

[7] Hoefnagels H F, Wu D, de With G, and Ming W 2007 Langmuir 2313158

[8] Gao L and McCarthy T J 2006 Langmuir 225998

[9] Brown J A 2011 North Carolina School of Science and Mathematics

[10] Leng B, Shao Z, de With G, and Ming W 2009 Langmuir 252456

[11] Hayn R A, Owens J R, Boyer S A, McDonald R S, and Lee H J 2011 J Mater Sci 462503

[12] Liu Y, Xin J H, and Choi C H 2012 Langmuir 2817426

[13] Tuteja A, Choi W, Mabry J M, McKinley G H, and Cohen R E 2008 PNAS 10518200

[14] Xue C H, Jia S T, Zhang J, and Ma J Z 2010 Sci. Technol. Adv. Mater. 11033002

[15] Dawood M K et al 2011 Langmuir 274126

[16] Bittoun E and Marmur A 2012 Langmuir 2813933

[17] Cassie A B D and Baxter S 1944 Trans. Faraday Soc. 50546

[18] Quere D 2008 Annul. Rev. Mat. Sci 3871

[19] Lafuma A and Quere D 2003 Nature Materials 2457

[20] Bico J, Marzolin C, and Quéré D 1999 Europhysics Letters 47220

[21] Choi W et al 2009 Adv. Mater. 212190

[22] Epstein A K, Wong T S, Belisle R A, Boggs E M, and Aizenberg J 2012 PNAS 10913182

[23] Kim P et al 2012 ACS Nano 66569

[24] Stone H A 2012 ACS Nano 6, p. 6536

[25] Anand S, Paxson A T, Dhiman R, Smith J D, and Varanasi K K 2012 ACS Nano 610122

[26] Rykaczewski K, Anand S, Subramanyam S B, and Varanasi K K 2013 Langmuir 295230

[27] Smith J D et al 2013 Soft Matter 91772

[28] Kim P, Kreder M J, Alvarenga J, and Aizenberg J 2013 Nano Letters 41793

[29] Yao X et al 2013 Nature Materials 12529

[30] Daniel D, Mankin M N, Belisle R A, Wong T S, and Aizenberg J 2013 Applied Physics Letters 102231603

[31] Nakajima A, Hashimoto K, and Watanabe T 2001 Monatshefte fur Chemie 13231

[32] Miwa M, Nakajima A, Fujishima A, Hashimoto K, and Watanabe T 2000 Langmuir 16 5754

[33] Yoshimitsu Z, Nakajima A, Watanabe T, and Hashimoto K 2002 Langmuir 185818

[34] Michielsen S and Lee H J 2007 Langmuir 236004

[35] Stober W, Fink A, and Bohn E 1968 J Coll Int Sci 2662

[36] Fadeev A Y and McCarthy T J 1998 Langmuir 145586

[37] Witucki G L 1993 J Coatings Tech 6557

[38] AATCC Technical Manual 200983

[39] Jan E G 2009 Proceedings of the Symposium on Natural Fibres 3

[40] Fang X, Yu Z, Sun X, Liu X, and Qin F 2009 Front. Chem. Eng. China 397

[41] Liu L, Zhao J, Zhang Y, Zhao F, and Zhang Y J 2011 Coll Int Sci 358277 
[42] Jafari F and Farzaneh M 2011 Applied Physics A 102195

[43] Zhang X et al 2008 J Euro Ceram Soc 282177

[44] Nguyen T P N, Brunet P, Coffinier Y, and Boukherroub R 2010 Langmuir 2618369

[45] Korosl G and Kovats E 1981 J Chem Eng Data 26323 